\title{
Strates
}

STRATES Matériaux pour la recherche en sciences sociales

6 | 1992

La question de l'environnement : naissance d'un débat en Pologne

\section{Europe(s) rurale(s) ? Premiers éléments d'une bibliographie}

Marie-Laure Marduel

\section{OpenEdition}

Journals

Édition électronique

URL : http://journals.openedition.org/strates/3503

DOI : $10.4000 /$ strates.3503

ISSN : $1777-5442$

Éditeur

Laboratoire Ladyss

Édition imprimée

Date de publication : 31 mars 1992

ISSN : 0768-8067

Référence électronique

Marie-Laure Marduel, «Europe(s) rurale(s) ? Premiers éléments d'une bibliographie », Strates [En ligne], 6| 1992, mis en ligne le 22 novembre 2007, consulté le 08 septembre 2020. URL : http:// journals.openedition.org/strates/3503; DOI : https://doi.org/10.4000/strates.3503

Ce document a été généré automatiquement le 8 septembre 2020.

Tous droits réservés 


\title{
Europe(s) rurale(s) ? Premiers éléments d'une bibliographie
}

\author{
Marie-Laure Marduel
}

Histoire

1 ASH Timothy Gaton, La chaudière ; Europe centrale, 1980-1990, Paris, Gallimard, 1990, $446 \mathrm{p}$.

2 BAECKE Antoine de (ss. la direction de), Une histoire de la démocratie en Europe, Paris, Éditions Le Monde/La Découverte, 1991, 410 p.

BEHAR Pierre, L'Autriche-Hongrie, idée d'avenir; permanences géopolitiques de l'Europe centrale et balkanique, Paris, PUF, 1991, 192 p.

4 BLUM Jérôme, The end of the Old Order in rural Europe, Princeton, New-Jersey, Prinston University Press, 1978, XI-505 p.

5 BURGUIERE André, KLAPISH-ZUBER Christiane, SEGALEN Martine, ZONABEND Françoise (dir.), Histoire de la famille, Paris, A. Colin, 1986, 2 vol.

6 CARPENTIER Jean, LEBRUN Francis et alii, Histoire de l'Europe, Préface de René Rémond, Paris, Seuil, 1992, 620 p. tabl.

7 CORM Georges, L'Europe et l'Orient; de la balkanisation à la libanisation, histoire d'une modernité inaccomplie, Paris, La Découverte, 1989, 384 p. DION Roger, Essai sur la formation du paysage rural français, Neuilly-sur-Seine, G. Durier, $1981,168 \mathrm{p}$. DION Roger, Les frontières de la France, Paris, Le Seuil, 1947. DION Roger, Le paysage et la vigne ; essai de géographie historique, Paris, Payot, 1990, 294 p. DION Roger, Histoire de la vigne et du vin en France; des origines au XIX $X^{e}$ siècle, Paris, Flammarion, 1990 (première édition, 1959), 768 p. DREYFUS François-Georges, L'Allemagne contemporaine, 1815-1900, Paris, PUF, 1991, $624 \mathrm{p}$.

13 DUBY Georges), WALLON Armand, (dir), Histoire de la France rurale, Paris, Seuil, 1975-1976, 4 tomes. 
DUBY Georges, Guerriers et paysans, $\mathrm{VI}^{e}-\mathrm{XII}{ }^{e}$ siècle, Premier essor de l'économie européenne, Paris, Gallimard, 1977, 308 p. ( $2^{e}$ éd. 1984).

DUROSELLE Jean-Baptiste, L'idée d'Europe dans l'histoire, Préface de Jean Monnet, Paris, Denoël, 1965, 347 p.

DUROSELLE Jean-Baptiste, L'Europe de 1815 à nos jours, Vie politique et relations internationales. $2^{e}$ éd. mise à jour, Paris, PUF, 1967, VIII + 399 p., cartes. ( $1^{\text {re }}$ éd. 1964).

DUROSELLE Jean-Baptiste (dir), L'Europe, histoire de ses peuples, Paris, Perrin, 1990, 423 p. index.

FOUCHER Michel, Fronts et frontières; un tour du monde géopolitique, Paris, Fayard, 1988, 446.

GELLNER Ernest, Nations et nationalismes, Paris, Payot, 1989, 206 p., (Bibliothèque historique Payot).

GLACKEN Clarence J., Traces on the Rhodian Shore; Nature and Culture in Western Throught from ancient times to the end of the eighteenth Century, Berkeley, Los Angeles, London, 1990, $763 \mathrm{p}$.

GOODY Jack, THIRSK E.-P., THOMSON E.-P. (eds.), Family and Inheritance; rural Society in Western Europe, 1200-1800, Cambridge, Cambridge University Press, 1983.

GROSSER Alfred (dir.), Les pays d'Europe occidentale, Paris, La Documentation française, 1988 (Notes et études documentaires, $n^{\circ}$ 4860-61/1988.)

GUIOMAR Jean-Yves, La Nation entre l'histoire et la raison, Paris, La Découverte, 1990, 204 p. (Coll. « Armillaire»)

LAUNAY Michel, Le syndicalisme en Europe, Paris, Imprimerie nationale, 1991, 504 p.

L'ÉCOTAIS Yann de, Naissance d'une nation, Paris, Grasset, 1990, 221 p.

LEQUIN Yves, MARILLARD J., L'Europe occidentale au $x x^{e}$ siècle, Nouvelle édition mise à jour, Paris, Masson, 1975, 118 p. $1^{\text {re }}$ édition, Masson, 1970, (Dossiers d'histoire.)

LEQUIN Yves, La mosaïque France; histoire des étrangers et de l'immigration, Préface de Pierre GOUBERT, Paris, Larousse, 1988, 480 p, Bibliogr. Index (Coll. «Mentalités vécues et représentations »).

LEWIN Moshe, La paysannerie et le pouvoir soviétique, 1928-1930, Préface de Roger Portal, Paris, La Haye, Mouton \& Co, 1966, 480 p.

LEWIN Moshe, Le dernier combat de Lénine, Paris, Ed. de Minuit, 1967, 173 p.

MENDES-SARGO Emmanuel, Thomas Munzer, les paysans et le communisme, Essai de sociologie sur la guerre des paysans en Allemagne (1525), Thèse de doctorat de sociologie, Nanterre, décembre 1986, $383+232 \mathrm{p}$.

MORIN Edgar, Penser l'Europe, Paris, Gallimard, 1989, 219 p.

NEWBY, BELL, ROSE, SAUNDERS, Property, Paternalism an Power; Class Control in rural England, London Hutchinson \& Co, 1978, 432 p.

NOIRIEL Gérard, Le creuzet français ; histoire de l'immigration XIX ${ }^{e}-\mathrm{XX}{ }^{e}$ siècles, Paris, Le Seuil, 1988, 437 p. (L'univers historique.)

NOUZILLE Jean, Histoire de frontières; l'Autriche et l'Empire ottoman. Préface de Jean Béranger, Paris, Éditions Berg international, 1991, 262 p.

POMIAN Krysztof, L’Europe et ses nations, Paris, Gallimard, 1989 (Coll. « Le débat »). 

$192 \mathrm{p}$.

Ethnologie $127 \mathrm{p}$ $1990,350 \mathrm{p}$.

SALT John, CLOUT Hugh, Migrations in Post-War Europe; geographical essays, London, Oxford University Press, 1976, 228 p., Notes, bibliogr. Index.

SCARDIGLI Victor, L'Europe des modes de vie, Paris, CNRS, 1985, 140 p.

SZUCS Jano, Les Trois Europes, Préface de Fernand Braudel, Paris, L’Harmattan, 1985,

WALL R., ROBIN J., LASLETT P. (eds), Family Froms in Historic Europe, Cambridge, Cambridge University Press, 1983.

WEBER Eugen, Une histoire de l'Europe, hommes, cultures et sociétés de la Renaissance à nos jours, Trad. de l'anglais par Philippe Delamare, Paris, Fayard, 1986. 2 vol.

WEBER Eugen, La fin des terroirs. La modernisation de la France rurale, 1870-1914, Paris, Fayard, Éditions Recherches, 1983, 839 p.

WRIGHT Gordon, L'Europe en Guerre, 1939-45, Trad. par Marthe Blinoff, Paris, A. Colin, 1971, 260 p. Cartes.

«Les marches de la Russie », Hérodote, 54-55, 4 e trimestre 1990, Paris, La Découverte,

«En Europe, les nations », Terrain, 17, octobre 1991, 166 p.

« Histoire, politique, société », Le Débat, 63, janvier-février 1991, Paris, Gallimard, 1991,

ANDORKA Rudolph, FARAGO Tamas, « Pre-industrial Household structure in Hungary », in, Family forms in Historic Europe / R. Wall, J. Robin, P. Laslett (eds), Cambridge, Cambridge University Press, 1983, p. 281-307.

AUGUSTINS Georges, La perpétuation des groupes domestiques dans les sociétés paysannes européennes, Nanterre, Paris-X, 1986, 2 tomes, $451+527$ p., Thèse, Ethnologie, Paris-X, 1986 (Directeur de thèse, Henri MENDRAS).

AUGUSTINS Georges, Se perpétuer, Paris, Lab. de sociologie et d'ethnologie comparative, Paris X-Nanterre, Société d'ethnologie, 1989, $434 \mathrm{p}$.

AUGUSTINS Georges, « Esquisse d'une comparaison des systèmes de perpétuation des groupes domestiques dans les sociétés paysannes européennes ", Archives européennes de sociologie, XXIII, 1982, p. 39-69.

BERKNER, L.K., MENDELS F., « Inheritance Systems, Family Structure and Demographic Patterns in Western Europe, 1700-1900 », in TILLY Charles (ed.), Historical Studies in Changing Fertility, Princeton, Princeton University Press, 1976.

BYRNES Robert F., Communal families in the Balkans; The Zadruga, Notre-Dame, Indiana, University of Indiana Press, 1976.

CAMPBELL J.K., Honor, Family and Patronage, Oxford, Oxford Clarendon Press, 1964.

CLOUT Hugh, «Land-use Change in finistere during the eighteenth and nineteenth centuries », Études rurales, LXXIII, 1979, p. 69-96.

CUISENIER Jen (dir.), Le cycle de la vie familiale dans les sociétés européennes, Paris, Mouton, $1977,494 \mathrm{p}$.

CUISENIER Jean, Problèmes de développement économique dans les pays méditerranéens, Actes du colloque international de Naples, 28.10, 2.11.1962. Préface de R. Aron, La Haye, Paris, Mouton \& Co, 1963, 438 p. 

sociales, Paris, Laboratoire d'anthropologie so
Bibliogr. (Études et documents balkaniques; 1 ). p. 3-20. University Press, 1972. p. 240-277. pénétration capitaliste, Paris, CNRS, 1969, 254 p. 1970, p. 99-114.

Structures territoriales et identités culturelles Paris, 1987, 290 p., tabl. (Coll. « Recherches ») «Cahiers libres ») Paris, L'Harmattan, août 1991, 190 p. Th. Pangalos, Paris, L'Harmattan, 1989.

CUISENIER Jean, Ethnologie de l'Europe, Paris, PUF, 1990, 126 p. (Que sais-je ?)

GOODY Jack, "Strategies of Heirship ", Comparative Studies in Society and History, XV-1,

GOODY Jack, «Inheritance, Property and Women, some comparative Perspectives », in GOODY Jack, THIRSK E.P., THOMSON E.P. (eds), Family and Inheritance. Rural Society in Western Europe 1200-1800, Cambridge, Cambridge University Press, 1983.

HERITIER Françoise, L'exercice de la parenté, Paris, EHESS/Gallimard/Le Seuil, 1981.

LASLETT Peter, WALL Richard, Household and Family in Past Time, Cambridge, Cambridge

PFEIFFER Gottfried, «The Quality of Peasant Living in Central Europe, in W-L THOMAS (ed.), Man's role changing the Face of the Earth, Chicago, Chicago University Press, 1956,

PINA CABRAL Jono de, «L'héritage du Maine, repenser les catégories descriptives dans l'étude de la famille en Europe ", Ethnologie française, XIX, 1989, 4, p. 329-340.

ROGERS Susan, « Female Forms of Power and the Myth of male dominance, a model of female/male interaction in peasant Society ", American Ethnologist, II-4, 1975, p. 727-756.

STAHL Henri, Ethnologie de l'Europe du sud-est, une anthologie, Paris, La Haye, École Pratique des Hautes Études, 1974, 312 p., Ind, (Textes de sciences sociales); 12.

STAHL Henri, Les anciennes communautés villageoises roumaines; asservissement et

STAHL Henri, Sociétés traditionnelles balkaniques, contribution à l'étude des structures sociales, Paris, Laboratoire d'anthropologie sociale; document ronéoté, 1979, 258 p.

TODD Emmanuel, L'invention de l'Europe, Paris, Seuil, 1990, 540 p.

WOLF Eric, "The Inheritance of Land among Tyrolese Peasants ", Anthropologica, XII,

CAIRE Guy, DELORME Robert, "L'Europe, quel espace social ?", Les Cahiers de la FEN,

DOMENACH Jean-Marie, Europe, le défi culturel, Paris, La Découverte, 1990, 156 p. (Coll.

"L'Est européen, sociétés à refonder, espaces à reconfirmer ", Espaces et sociétés, 64,

FRANCE-INSEE, La France et ses régions, Paris, INSEE, 1990, $150 \mathrm{p}$.

GEORGE Pierre, Fin de siècle en Occident, déclin ou métamorphose ?, Paris, PUF, 1982, 190 p.

GUERY Gabriel, La dynamique de l'Europe sociale, Paris, Dunod, 1991, 297 p.

LACOSTE Yves (dir.), Géopolitique des régions françaises, Paris, Fayard, 1986, 3 vol.

MAPPA Sophia dir, La CEE, chance ou contrainte pour la transformation sociale, Préface de

MENDRAS Henri, Sociétés paysannes; éléments pour une sociologie de la paysannerie, Paris, A. Colin, 1976. 

1978. libres/ essais ». $741 \mathrm{p}$. 1986, $39 \mathrm{p}$. $144 \mathrm{p}$.

MENDRAS Henri, SCHNAPPER Dominique (dir.), Six manières d'être européen, Paris, NRF/ Gallimard, 1990, 293 p. (Bibliothèque des sciences humaines.)

MENDRAS Henri, REILLIER Frédéric, Trois cent quarante millions d'Européens, Paris, Ramsay, 1990, 192 p., Bibliogr. Index.

PRATE Alain, Quelle Europe?, Préf. de Raymond Barre et Jacques Delors, Paris, Commentaires Julliard, 1991, $436 \mathrm{p}$.

RAMBAUD Placide, «L'utopie communautaire et l'idéologie de la nation », Projet, maijuin 1989, p. 53-63.

RADVANYL J., L'URSS, Régions et nations, Paris, Masson, 1990, 293 p.

RAVIS-GIORDANI Georges, "Espaces et groupes sociaux, organisation objective et appréhension symbolique ", in Pieve e Paesi, communautés rurales corses, Paris, CNRS,

SELLIER André, SELLIER Jean, Atlas des peuples d'Europe centrale, Paris, La Découverte, septembre 1991, $192 \mathrm{p}$.

«En Europe, les nations », Terrain, 17, octobre 1991, 166 p.

"L'identité, un mythe-refuge", Revue des sciences sociales de la France de l'est, 18, Strasbourg, Université des sciences humaines, 1991, $197 \mathrm{p}$.

L'identité régionale ; l'idée de la région dans l'Europe du sud-ouest, Paris, CNRS, 1991, 400 p.

Pierre Héritier, Dominique Taddei, Jacques Ion, Hugues Bertrand, Anne-Marie Grozelier, Les enjeux de l'Europe sociale, Paris, La Découverte, 1991, 170 p., coll. «Cahiers

« Pouvoirs, territoires, identités dans les sociétés rurales européennes » (mélanges) par Marie- Claude Maurel, Michel Sivignon, Michel Drain et al., Espace rural (20), 1990, 150 p. (Montpellier, Publications de l'Université Paul Valéry.)

Organisations professionnelles et vie politique

BIBES Geneviève, MOURIAUX René, Les syndicats européens à l'épreuve, Paris, Presses de la FNSP, 1990, $291 \mathrm{p}$.

BIHR Alain, Du grand soir à l'alternative, le mouvement ouvrier en crise, Paris, Éditions ouvrières, 1991, 228 p., Coll. « Portes ».

2 CENTRE D'ÉTUDES DE LA VIE POLITIQUE FRANÇAISE (CEVIPOF), Les organisations syndicales et professionnelles en Europe, Paris, FNSP, 1989, 121 p., (Cahiers du CEVIPOF, 5).

COMITÉ ÉCONOMIQUE ET SOCIAL, L'agriculture européenne et ses organisations, Luxembourg, Office des publications officielles des Communautés européennes, 1984,

4 COULOMB Pierre, DELORME Hélène, Les mutations de l'agriculture dans les pays industriels. II. Les agriculteurs et leurs organisations, Paris, La Documentation française,

DELORME Hélène, TAVERNIER Yves, Les paysans français et l'Europe, Paris, A. Colin, 1969,

DEVIN Guillaume (dir.), Syndicalisme, dimensions internationales, Paris, Éditions européennes Erasme, $420 \mathrm{p}$. 

p. 3-11. 1989, 383 p.

INSTITUT D'ÉTUDES EUROPÉENNES, Un défi pour la Communauté européenne, les bouleversements à l'est et au centre du continent, Bruxelles, Éditions de l'université de Bruxelles, 1991, 297 p. (Études européennes.)

LAUNAY Michel, Le syndicalisme en Europe, Paris, Imprimerie nationale, 1991, 504 p.

LEMOINE Nathalie, Le syndicalisme agricole français et l'élargissement de la CEE à l'Espagne et au Portugal, dactyl. mém. DEA, Sc. polit., Paris-I, 1986 (dir. M. MERLE).

Les agriculteurs et la politique/ss. la direction de Pierre Coulomb, Hélène Delorme, Bertrand Hervieu, Marcel Jollivet, Philippe Lacombe, Paris, FNSP, 1990, 594 p. Index.

Où va l'Est? Actes du colloque de la Sorbonne du 20 février 1990. «Réformes politiques et économies de transmission ", Journal des élections, Paris, Libération, 1990, 114 p.

Pour une alternative verte en Europe, Pierre Juquin, Carlo Antunes, Penny Kemp, Isabelle Stengers, Wilfred Telkamper, Frieder Otto Wolf, Paris, La Découverte, 1990, 128 p. (Coll. Cahiers Libres.)

Économie rurale

ALLAYA M., LABONNE M., PAPAYANNAKIS M., Les échanges agroalimentaires méditerranéens, enjeu mondial, Montpellier, CIHEAM-IAMM, 1988, 307 p.

ARNAUD Charles, "Le vin et l'organisation commune de marché entre Paris et Bruxelles, un dialogue parfois difficile", Économie rurale, n 204. juillet-août 1991,

BARTHE René, L'Europe du vin. Vingt-cinq ans d'organisation communautaire du secteur vitivinicole (1962-1987), Préface d'Edgar Faure, Montpellier, INRA/ESR, 1988 et Paris, Cujas,

BARTHEZ Alice, Famille, travail et agriculture, Paris, Economica, 1982, 189 p. bibliogr.

BERGMANN Denis, A Future for European Agriculture. L'avenir de l'agriculture européenne, Londres, 30 mai 1985. Paris, INRA-ESR, 1985.

BOURGET Bernard, "L'agriculture européenne et l'évolution des marchés mondiaux ", Agriscope (11), p. 37-43.

BUTAULT J-P, Spécialisation et avantages comparés des agricultures européennes, séance 3 , politiques économiques et déséquilibres macroéconomiques. Colloque "Stratégies internationales et intégration européenne », 21-22 septembre 1987, $24 \mathrm{p}$.

BUTAULT Jean-Pierre, CYYNCYNATUS M., HASSAN Daniel, «Les avantages comparés des agricultures européennes ", Économie rurale, n 197, mai-juin 1990, p. 15-21.

CHARVET Jean-Paul, Modernisation des filières agricoles dans le contexte d'une concurrence internationale accrue / J.-P. Charvet, G. Dorel, V. Rey et al., Paris, Commissariat général du plan, Centre de géographie rurale de Paris-X, octobre 1987, $97+119$ p.

CHARVET Jean-Paul, La guerre du blé, Paris, Economica, 1990, Coll. «Cyclope », 103 p.

CHARVET Jean-Paul, Les greniers du monde, Paris, Economica, 1986, Coll. "Économie agricole et agroalimentaire », $368 \mathrm{p}$.

COMMISSION DES COMMUNAUTÉS EUROPÉENNES, La situation de l'agriculture dans la Communauté. Rapport publié chaque année.

COMMISSION DES COMMUNAUTÉS EUROPÉENNES, Cinq années de progrès. Coordination de la recherche agricole dans la Communauté économique européenne, 1984-1988, 
Luxembourg, Office des publications officielles des communautés européennes, 1985, $37 \mathrm{p}$.

116 COMMISSION DES COMMUNAUTÉS EUROPÉENNES, Les exploitations agricoles des zones défavorisées et de montagne de la Communauté, Luxembourg, Office des publications officielles des communautés européennes, 1989, $129 \mathrm{p}$.

117 COULOMB Pierre), DELORME Hélène, Les mutations de l'agriculture dans les pays industriels. I. La crise des marchés internationaux et les réponses institutionnelles, Paris, La Documentation française, 1986, $35+39 \mathrm{p}$.

118 DELORME Hélène, Le contentieux agricole CEE-États-Unis; le cas des céréales in, BOURRINET J. (dir.), Les relations Communautés européennes-États-Unis, Paris, Economica, 1987.

119 DREVET Jean-François, 1992-2000, les régions françaises entre l'Europe et le déclin, Paris, Ed. Soufflet, avril 1988, $250 \mathrm{p}$.

120 FRANCE-INSEE, L'agriculture dans la CEE, tome 1, Vingt-cinq ans de production. Jean Leguennec, Paris, INSEE, 1987. (Collection de l'INSSEE ; E 104.)

121 FRANCE-INSEE, L'agriculture dans la CEE, tome 2, Emploi et activités. Eric Vert, Paris, INSEE, 1987. (Collection de l'INSEE ; E. 108.)

FRANCE-INSEE, L'agriculture dans la CEE, tome 3, Coûts de production. J.-P. Butault, D. Carles, D. Hassan, E. Reignier, Paris, INRA, INSEE, 1988. (Collections de l'INSEE; E 112.)

123 FRANCE-INSEE, Atlas des régions de l'Europe verte. L'agriculture dans la CEE, tome 4 / par Nicole Duval et Eric Vert, Amiens, INSEE, 1990, 88 p., tabl., cartes, Bibliogr. Annexes. (INSEE Résultats, $n^{\circ} 54$, Système productif, $n^{\circ} 24$, mars 1990.)

124 FRANCE. Ministère de l'Agriculture et de la Forêt, GRAPH AGRI EUROPE, Paris, SCEES, 1989, 79 p. Index.

125 FRANCE. Ministère de l'Agriculture - SCEES, Classification révisée des exploitations agricoles (OTEX-CDEX « $82 »)$, Collections de statistique agricole. Étude $n^{\circ} 282$, avril 1989, Paris, SCEES, $1989,89 \mathrm{p}$. GERBAUX F.), MULLER P., «La pluriactivité agricole dans les nouvelles stratégies paysannes », p. 203-209 in, La pluriactivité dans les familles agricoles, Paris, ARF, 1984. GRALL Jacques), LEVY Bertrand Roger, La guerre des semences. Quelles moissons? Quelles sociétés ?, Paris, Fayard, 1985, 410 p. HULOT Jean-François, LOYAT Jean, LEGENDRE Janine, « Agriculture d'entreprise au nord de la Communauté et familiale au sud », Cahiers de statistique agricole, 5, mars 1991, p. 41-49. LACOMBE Philippe, "Les exploitations agricoles, modèle ou réalité », p. 88-101 in, L'agriculture dans le monde rural de demain, à nouveaux enjeux, droit nouveau, Paris, La Documentation française, 1986, $160 \mathrm{p}$. NEVEU A., Agriculture, économie de l'agriculture française en Europe, forces et faiblesses, Paris, Dunod, 1991, 192 p. Bibliogr.

131 PISANI Edgar, "L'Europe agricole doit sortir de sa forteresse ", Économie et finances agricoles, supplément au numéro de juin-juillet 1983.

132 RATTIN Solange, «La structure des exploitations agricoles dans l'Europe des Dix en 1985 », Cahiers de statistique agricole, 1/6, 1988. 
RATTIN Solange, "Quatre types d'agriculture en France... trois groupes de pays en Europe », Cahiers de statistique agricole, 6/6, 1988.

RATTIN Solange, «Une agriculture européenne à trois vitesses ", Cahiers AGRESTE, 3, septembre 1990.

SARRIS Alexander, "Agriculture européenne, marchés internationaux, croissance des PVD et sécurité alimentaire ", Économie rurale, 205, sept.-oct. 1991, numéro spécial, L'agriculture européenne à la recherche de nouvelles stratégies, p. 5-15.

SIDJANSKI Dusan, AYBERK Ural (eds), L'Europe du sud dans la Communauté européenne, Genève, Institut universitaire d'études européennes de Genève, 1990, 280 p.

SNEESSENS Jean-François, "La cohérence de l'agriculture européenne ». Économie rurale, 191, mai-juin 1989, p. 24-28.

Industries agroalimentaires et alimentation

CLUB DE BRUXELLES, L'industrie agroalimentaire dans le marché unique, Bruxelles, Club de Bruxelles, 1991, $215 \mathrm{p}$.

HENZE A., ZEDDIES Dr. J., «Les adaptations de l'agriculture de la CEE, programmes communautaires, effets économiques et analyse coût-avantage", Économie rurale, $\mathrm{n}^{\circ} 189$, janvier-février 1989, p. 35-41.

KROLL Jean-Christophe, «Choc des nations et compétitivité dans l'agroalimentaire européen », Économie rurale, 196, mars-avril 1990, p. 16-23.

NEFUSSI J., Les industries agroalimentaires en France, croissance et financement, 1950- 1985 sur l'intégration financière et la dynamique industrielle, 1987, 2 vol. Th. Sc. éco., Paris-X, 1987 (Dir. F. RENVERSEZ.)

ROBLEZ M.-J., L'HARDY P., TROGNON A. (dir.), Comparaison internationale de la demande de produits alimentaires, Paris, INRA/ESR, CREDOC, Commissariat général du plan, direction générale de la concurrence, de la consommation et de la répression des fraudes, octobre 1987, 130 p. (Collection des rapports ; 22.)

TRAILL B., Prospects for the European Food System, Paris, Agro-Lavoisier, 1989, 250 p.

"La compétitivité dans l'agroalimentaire européen", Économie rurale, 195, janvierfévrier 1990, Versailles, SFER, 1990, 56 p.

«La réponse des IAA européennes au défi des technologies». Colloque de l'Institut Pasteur, Paris, 14 et 15 mars 1989, Paris, Lavoisier, 1989, 258 p, Bibliogr.

Espace rural et société rurale

ASSIER-ANDRIEU Louis, "Le droit et les paysans ", Études rurales (103-104), juil.-déc. 1986, p. 9-13.

7 AUDEMA M., «Le fait rural européen », Bulletin du Conseil général du GREF (Génie rural des eaux et forêts), juillet 1991, 30, $11 \mathrm{p}$.

BODIGUEL Maryvonne, LOWE Philippe, Campagne française, campagne britannique, un carrefour des sciences sociales, Paris, L'Harmattan, 1990, 355 p.

BULLER Henry, WHRIGHT S., Rural Development, problems and practices, Aldershot, Great Britain, Brookfield, Vermont USA, Gower Publishing Co, 1990, 249 p.

BUTTEL F.H., NEWBYH. (eds), The Rural Sociology of Advanced Societies, Critical Perspectives, London, Croom Helm, 1980. 
COMMISSION DES COMMUNAUTÉS EUROPÉENNES, «L'avenir du monde rural». Communication de la Commission du Parlement européen, Bulletin des Communautés européennes, supplément 4/88, 71 p. (Journal officiel)

GREP - «L'Europe rurale à l'heure des choix », Pour, 115-116, Toulouse, Éditions Privat, $1988,186 \mathrm{p}$.

LORVELLEC Louis, Droit rural, Paris, Masson, 1987, 568 p.

MEDEIROS Fernando, Espaces ruraux et dynamiques sociales en Europe du sud, Braga, XIII congrès européen de sociologie rurale.

OCDE, Formulation de la politique rurale ; nouvelles tendances, Paris, OCDE, 1988, 197 p.

"Quel avenir pour l'agriculture et le monde rural?", Économie rurale, 202-203, marsjuin 1991, Versailles, SFER, 1991, 124 p. (Session des 5 et 6 juin 1990, organisé par Isabelle BOUSSARD (FNSP) et Bernard WOLFER (INRA);

Environnement

BODIGUEL Maryvonne (dir.), Produire et préserver l'environnement, quelles réglementations pour l'agriculture européenne?, Paris, L'Harmattan, 1990.

CHARPENTIER Jean (dir.), La protection de l'environnement par les communautés européennes, Préface d'André Rossinot, Paris, A. Pedone, 1989.

CNRS-PIREN, «Le droit de l'environnement ». Actes des journées de l'environnement du CNRS, 30 novembre-1 ${ }^{\text {er }}$ décembre 1988, Paris, CNRS-PIREN, 1990, 317 p.

COMMISSION DES COMMUNAUTÉS EUROPÉENNES, 1992. The environmental Dimension, Bruxelles, Commission des Communautés européennes, 1989.

COMOLET André, «Europe agricole. L'environnement sous contrat », Études foncières, 43, juin 1989, p. 5-9.

CORCELLE G., JOHNSON S., L'autre Europe "verte». La politique communautaire de l'environnement, Paris, Ed. Labor/Fernand Nathan, 1989.

ÉCOLE NATIONALE DU GÉNIE RURAL DES EAUX ET FORÊTS 5ENGREF), SECRÉTARIAT D'ÉTAT CHARGÉ DE L'ENVIRONNEMENT, La politique européenne de l'environnement, Paris, Romillat, 1990, $191 \mathrm{p}$.

Futuribles, $\mathrm{n}^{\circ}$ spécial « Prospective de l'environnement en Europe » (89), 1985.

GERMON J., Management Systems to reduce the impact of Nitrates, Paris, Agro-Lavoisier, 1989, $274 \mathrm{p}$.

KISS Alexandre, L'écologie et la loi ; le statut juridique de l'environnement, Paris, CNRS/ L'Harmattan, 1989, $391 \mathrm{p}$.

KISS Alexandre, Droit international de l'environnement, Paris, PEDONE, 1989.

LARRUE Corinne, "Protection des eaux et agriculture en France, Suisse et Belgique ", p. 208-217 in MATHIEU Nicole, JOLLIVET Marcel (dir.), Du rural à l'environnement; la question de la nature aujourd'hui, Paris, ARF/L'Harmattan, 1989.

NICOLLON A., SALLES J.-M., Enjeux économiques et technologiques des politiques de l'environnement en Europe. Fascicule II, La protection des cultures et de l'environnement dans la perspective de 1992. Rapport France. EHESS. CIRED, Paris, SRETIE, janvier 1990, 72 p. (Rapport pour le Groupe Prospective. Contrat STRETIE/MERE/7160.)

$70 \mathrm{OCDE}$, Politiques de l'agriculture et de l'environnement; possibilités d'intégration, Paris, Éditions de l'OCDE, 1989. 
PRIEUR Michel (dir.), Europe des régions et environnement, Limoges, Publications de la Faculté de droit et de sciences économiques de l'université de Limoges, 1989, $324 \mathrm{p}$. ROELANTS DU VIVIER F., HANNEQUART J.-P., Agriculture européenne et environnement, un avenir fertile, préface de Jacques Delors, Paris, Éditions Sang de la Terre, 1987, 302 p.

ROMI Raphaël, L'Europe et la protection juridique de l'environnement, préface de Carlo Ripa di Meana, Commissaire européen pour l'environnement, la sécurité nucléaire et la protection civile, Paris, LITEC, 1990.

SCHNEIDER G., CORNAERT M.-H. (dir.), L'État de l'environnement dans la Communauté européenne, 1986, Luxembourg, OPCE, 1987, 383 p.

THIEBAUT Luc, L'Europe de l'environnement et l'agriculture française. Rapport France INRA/ENSAA, Dijon, 1987, 4 p. (Document intermédiaire)

Politique agricole

AFIP, (matériaux pour la formation, L'Europe verte (1), Histoire et rouages, décembre 1984 (2), À la croisée des chemins, juin 1985.

BAZIN G., «Nouvelles orientations de la politique agricole commune et zones défavorisées ». Communication au séminaire « Agricultures régionales, concurrence et politiques économiques »; France, Italie, Espagne ; Montpellier, 25-27 avril 1988, 15 p.

BERGMANN D., «La politique agricole commune ; crise et remèdes ", Économie rurale (143), 1981, p. 5-15.

CARFANTAN Jean-Yves, L'Europe verte sous influence ; l'heure du choix, Paris, Seuil, 1985, $282 \mathrm{p}$.

CEE, La politique agricole de la Communauté européenne, Luxembourg, Office des publications officielles des Communautés européennes, 1982.

COULOMB Pierre, DELORME Hélène, «L'agriculture française à l'heure de la croissance zéro ", Économie rurale (163), sept.-octobre 1984. p. 41-47.

ÉCONOMIE RURALE, numéro spécial (163), septembre-octobre 1984, «Crise de la PAC et PAC de la crise », Versailles, SFER, 1984.

GORDON K., «Transition Stratégies for agricultural Policy Reforms », European Review of Agricultural Economics, vol. 15-4, 1988, p. 1-490.

GOUSSAULT Yves (dir.), Politique agricole nationale et communautaire. Conséquences sur l'agriculture régionale. Étude réalisée pour l'Établissement public régional ProvenceAlpes-Côte d'Azur, Aix-Marseille, Université de Provence. Lab. de sociologie de l'industrialisation et du développement, juillet 1977, 283 p. Annexes.

KROLL Jean-Christophe, Agriculture, changer de politique, Paris, Syros, 1990, 192 p., Coll. «Syros Alternative ».

KOESTER U., Policy Options for the Grain Economy of the European Community, implications for developping countries, Washington, IFPRO, 1982 (Research Report $n^{\circ} 35$. )

LABOUZ M.-F., Le système communautaire européen, Paris, Berger-Levrault, 1986, $350 \mathrm{p}$. (Mondes en devenir, XVIII, Manuel BL6.)

LAUGA Louis, La politique agricole commune sur les chemins de la nécessité. Rapport présenté par Louis Lauga au $37^{\mathrm{e}}$ congrès de la FNSEA, avril 1983.

MAHE L.-P., TAVERA C., "Bilateral Harmonization of EC and US agricultural Policies », European Review of Agricultural Economics, vol. 15, n 4, 1989, p. 327-348. 
MULLER Pierre, VALCESCHINI E., Vers une politique de l'exploitation rurale. Communication présentée au colloque « Diversification des modèles de développement rural ». 17-28 avril 1986, $30 \mathrm{p}$.

ORGANISATION DE COOPÉRATION ET DE DÉVELOPPEMENT ÉCONOMIQUE (OCDE), Politiques, marchés et échanges agricoles, Paris, OCDE, 1989, 354 p.

PHILIPPE Bernard, Politique agricole européenne et marchés mondiaux, Paris, Economica, 1975,286 p.

SERVOLIN Claude, "L'agriculture et l'Europe, la France dans la PAC», p. 128-194 in SERVOLIN Claude, L'agriculture moderne, Paris, Sseuil, 1989, 318 p, Bibliogr.

TRACY Michel, L'État et l'agriculture en Europe occidentale. Crise et réponses au cours d'un siècle, Paris, Economica, 1986, 471 p. ill., cartes, Bibliogr. Index.

TRACY Michel, «Excédents et échanges, dilemmes européens », Économie rurale, 173, mai-juin 1986.

TRACY M., HODAC I. (dir.), Perspectives pour l'agriculture dans la Communauté européenne, Bruges, de Tempel, 1979.

TUBIANA Laurence, BECHE J.-M., La politique méditerranéenne des douze et les pays tiers méditerranéens, Montpellier, INRA/LEI, 1985, 31 p.

Politiques régionales

CONSEIL ÉCONOMIQUE ET SOCIAL, L'articulation des politiques européennes nationales et régionales d'aménagement du territoire, Avis et rapports du Conseil économique et social, 12, mai 1989, $123 \mathrm{p}$.

GASPERINI E., "Intégration européenne et accroissement des disparités régionales. La politique régionale de la CEE en question ", Économie et sociétés, 2, hors-série, 1989, p. 129-159.

KRAMER Ludwig, « La CEE, l'environnement, et la politique régionale », Revue du Marché commun, $\mathrm{n}^{\circ} 321$, novembre 1988, p. 539-553.

La politique régionale de la CEE, Paris, PUF, 1991, 126 p., Coll. « Que sais-je ?».

OBSERVATOIRE INTERRÉGIONAL DU POLITIQUE (OIP), Enquête 1988. Le fait régional. La Région et l'Europe. Rapport, Paris, OIP (FNSP/CNRS), 1988.

PLANQUES B., "Grand Marché unique européen et développement régional. Éléments de problématique ", Revue d'économie régionale et urbaine, I, 1990, p. 27-57.

RENCKIG., "La politique régionale communautaire, exemple d'une politique d'accompagnement du marché unique », Administration, 142, 15 janvier 1989, p. 122-126. ROMUS Paul, L'Europe régionale, Bruxelles, Éditions Labor, mars 1990, 150 p.

UHRICH René, Pour une nouvelle politique de développement régional en Europe, Préface de Pierre Pfimlin, Paris, Economica, 1983, IX, 196 p. 


\section{AUTEUR}

MARIE-LAURE MARDUEL

Ingénieur au Groupe de recherches sociologiques (CNRS/Univ. Paris-X) où elle gère une importante bibliothèque spécialisée sur les questions rurales et agricoles. 